\title{
Dome-and-dart T Waves and Hyperthyroidism - A Case Report
}

\author{
Matteo Parolin, Francesca Dassie, Eugenio De Carlo, Roberto Vettor and Pietro Maffei \\ Department of Medicine, Internal Medicine-3, University of Padova, Padova, Italy
}

DOI: https://doi.org/10.17925/EE.2020.16.1.69

W e briefly describe a case of a 31-year-old man with persistent hyperthyroidism, despite medical treatment with high dose methimazole. Twelve-lead 24-hour Holter monitoring showed bifid (or dome-and-dart) T waves and echocardiography revealed mild left ventricle dilatation. Hyperthyroidism was eventually treated with total thyroidectomy, and thereafter, T waves became normal and the left ventricle returned to normal dimensions. Hyperthyroidism should be considered among the differential diagnoses when T wave abnormalities are observed on electrocardiogram and when mild left ventricle dilatation is observed on an echocardiogram. The correction of hyperthyroidism can reverse these abnormalities.

\section{Keywords}

Hyperthyroidism, electrocardiogram, bifid T waves, Graves' disease, Holter ECG

Disclosures: Matteo Parolin, Francesca Dassie, Eugenio De Carlo, Roberto Vettor and Pietro Maffe have no financial or non-financial relationships or activities to declare in relation to this article.

Review Process: Double-blind peer review.

Acknowledgements: Editorial assistance for the preparation of this manuscript was provided by Alexandra Wennberg, PhD.

Compliance with Ethics: Informed consent was received from the patient involved in this case study. No identifying information or images have been included.

Authorship: All named authors meet the criteria of the International Committee of Medical Journal Editors for authorship for this manuscript, take responsibility for the integrity of the work as a whole and have given final approval for the version to be published.

Access: This article is freely accessible at

touchENDOCRINOLOGY.com @) Touch Medical Media 2020.

Received: 18 October 2019

Accepted: 29 November 2019

Published Online: 28 February 2020

Citation: European Endocrinology. 2020;16(1):69-71

Corresponding Author: Matteo Parolin,

Internal Medicine 3, University of Padova

Via Giustiniani 2, 35128, Padova, Italy.

E: matteoparolin@hotmail.it

Support: No funding was received in

the publication of this article.
Hyperthyroidism is a disease characterised by excess thyroid hormone synthesis and secretion from the thyroid gland. It is most frequently caused by Graves' disease and toxic nodular goiter. Systemic complications involving the respiratory, cardiac and gastrointestinal systems, as well as ocular manifestations, such as exophthalmos, can be caused by hyperthyroidism. Common cardiologic complications are atrial fibrillation, heart failure, pulmonary hypertension and angina pectoris. ${ }^{1}$ Here we present a case report of a young man affected by hyperthyroidism with bifid (or dome-and-dart) $\mathrm{T}$ waves on the electrocardiogram (ECG).

\section{Case report}

A 31-year-old man presented to a centre in April 2018 with excessive sweating, tremors, myalgias, and a 12-kg weight loss. These symptoms started about 3 months before first presentation. Graves' disease was diagnosed with laboratory tests that showed suppressed thyroid-stimulating hormone (TSH; <0.005 mUI/L [reference range 2.00-4.00 mUI/L]) and high free thyroxine 4 (FT4; $>99.9 \mathrm{pmol} / \mathrm{L}$ [reference range 10.3-23.2 pmol/L]). TSH-receptor antibodies were found to be elevated (22 UI/L, positive if $\geq 1 \mathrm{UI} / \mathrm{L}$ ). Thyroid ultrasonography showed an enlarged gland with increased vascularity. The patient was started on therapy with methimazole at a dose of $20 \mathrm{mg}$ per day (divided into two doses of $10 \mathrm{mg}$ ). Subsequent evaluations revealed persistent suppression of TSH, so the methimazole dose was increased to $30 \mathrm{mg}$ a day (divided into three equal doses) and prednisone $25 \mathrm{mg}$ daily was started.

He was referred to our clinic in November 2018 for persistent hyperthyroidism. Blood exams documented suppressed TSH and elevated free triiodothyronine (FT3) and FT4 (these exams were performed in another lab and are not available). He was compliant with his medications, but continued to complain of excessive sweating, tremors, myalgias and mild ophthalmopathy. A physical examination revealed enlarged, symmetric, soft thyroid and mild exophthalmos. Blood pressure was 120/80 mmHg, heart rate was 87 bpm and body weight was $73 \mathrm{~kg}$. No other physical abnormalities or electrolyte imbalances were detected. Medications for hyperthyroidism were adjusted to methimazole $40 \mathrm{mg}$ (divided into two doses), and $20 \mathrm{mg}$ of propranolol twice a day was started. Prednisone (25 mg daily) was continued. It was decided that either radioactive iodine therapy or total thyroidectomy would be the best treatment option. We discussed the benefits and risks of each strategy with the patient, and, finally, he chose the surgical approach, because he desired prompt control of the hyperthyroidism, and preferred to avoid radioactive exposure.

On 24 January 2019, he was seen by our surgeon, an expert in thyroidal surgery. At that time, laboratory tests revealed TSH $0.02 \mathrm{mUI} / \mathrm{L}$, FT4 $62.9 \mathrm{pmol} / \mathrm{L}$ (reference range $9.0-22.0 \mathrm{pmol} / \mathrm{L}$; a slight difference in reference ranges between difference labs can exist) and FT3 36.7 pmol/L (reference range 3.9-6.8 pmol/L) The dose of methimazole was further increased to $50 \mathrm{mg}$ per day (divided into two doses).

On 30 January 2019, he was admitted to our hospital. Laboratory exams continued to show hyperthyroidism (TSH $0.01 \mathrm{mUI} / \mathrm{L}, \mathrm{FT} 443.6 \mathrm{pmol} / \mathrm{L}$ and FT3 $15.1 \mathrm{pmol} / \mathrm{L}$ ). Propranolol was increased 
Figure 1: Electrocardiogram recorded at the presentation (active hyperthyroid status)

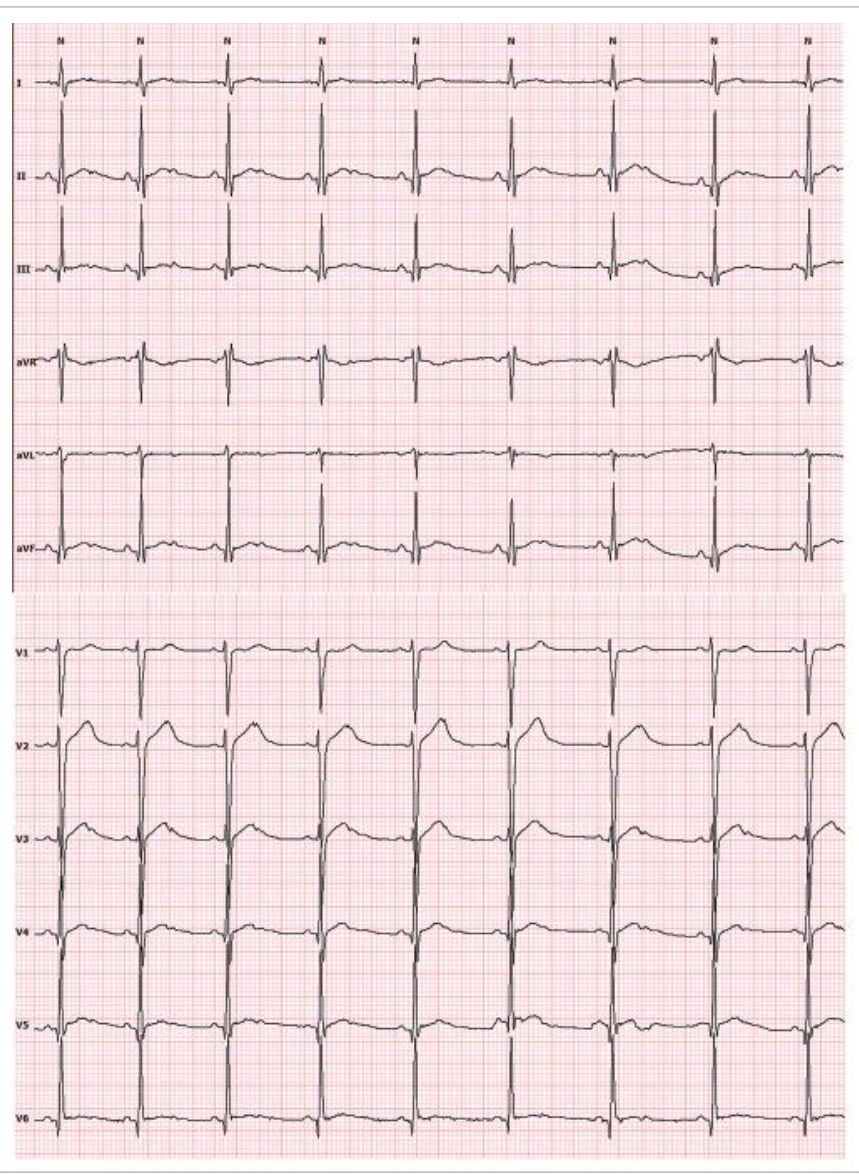

Electrocardiogram (ECG) showed sinus rhythm, heart rate of 75 beat per minute, ventricular axis $75^{\circ}, P Q$ segment $0.12 \mathrm{~s}$, Sokolov-Lyon criteria positive for left ventricular hypertrophy, diffuse dome and dart T waves, especially in leads D2, D3, aVF and from V2 to V5.

to $40 \mathrm{mg}$ three times per day and therapy with Lugol's solution (a solution of elemental iodine and potassium iodide given as a pre-operative treatment) was started. ${ }^{2}$ At admission, the 12-lead 24-hour Holter monitoring showed sinus rhythm, signs of left ventricular hypertrophy and diffuse ST-T abnormalities with initial dome and subsequent dart $T$ waves throughout the recording (Figure 1). The echocardiogram showed a mild dilatation of the left ventricle (end diastolic volume $79 \mathrm{~mL} / \mathrm{m}^{2}$ [reference range $54 \pm 10 \mathrm{~mL} / \mathrm{m}^{2}$ ], end systolic volume $32 \mathrm{~mL} / \mathrm{m}^{2}$ [reference range $21 \pm 5 \mathrm{~mL} / \mathrm{m}^{2}$ ], diastolic diameter $5.6 \mathrm{~cm}$ [reference range $5.0 \pm 0.4 \mathrm{~cm}]$ ), without other relevant findings. On 8 February 2019, the patient underwent total thyroidectomy. No complications occurred after the surgical procedure and thyroid hormone replacement therapy (L-thyroxine) was started.

After 1 month of L-thyroxine (at a dose of $50 \mathrm{mcg}$ per day), laboratory tests showed hypothyroidism with TSH (4.32 mUl/L) FT4 (6.0 pmol/L), FT3 (1.9 pmol/L), parathyroid hormone $(36.4 \mathrm{ng} / \mathrm{L}$ [reference range 6.5-36.8 ng/L]), calcium (2.4 mmol/L [reference range $2.1-2.6 \mathrm{mmol} / \mathrm{L}$ ]) and phosphorus (1.3 mmol/L [reference range $0.87-1.45 \mathrm{mmol} / \mathrm{L}$ ) . In addition, the Holter monitoring showed a normal sinus rhythm without ECG signs of left ventricular hypertrophy or abnormalities of ST-T waves (Figure 2). Moreover, the echocardiogram showed normal dimension of left ventricle (end diastolic volume $64 \mathrm{~mL} / \mathrm{m}^{2}$, end systolic volume $26 \mathrm{~mL} / \mathrm{m}^{2}$, diastolic diameter $5 \mathrm{~cm}$ ). For the mild hypothyroidism, L-thyroxine was increased to 75 mcg per day.
Figure 2: Electrocardiogram recorded after normalisation of hyperthyroidism

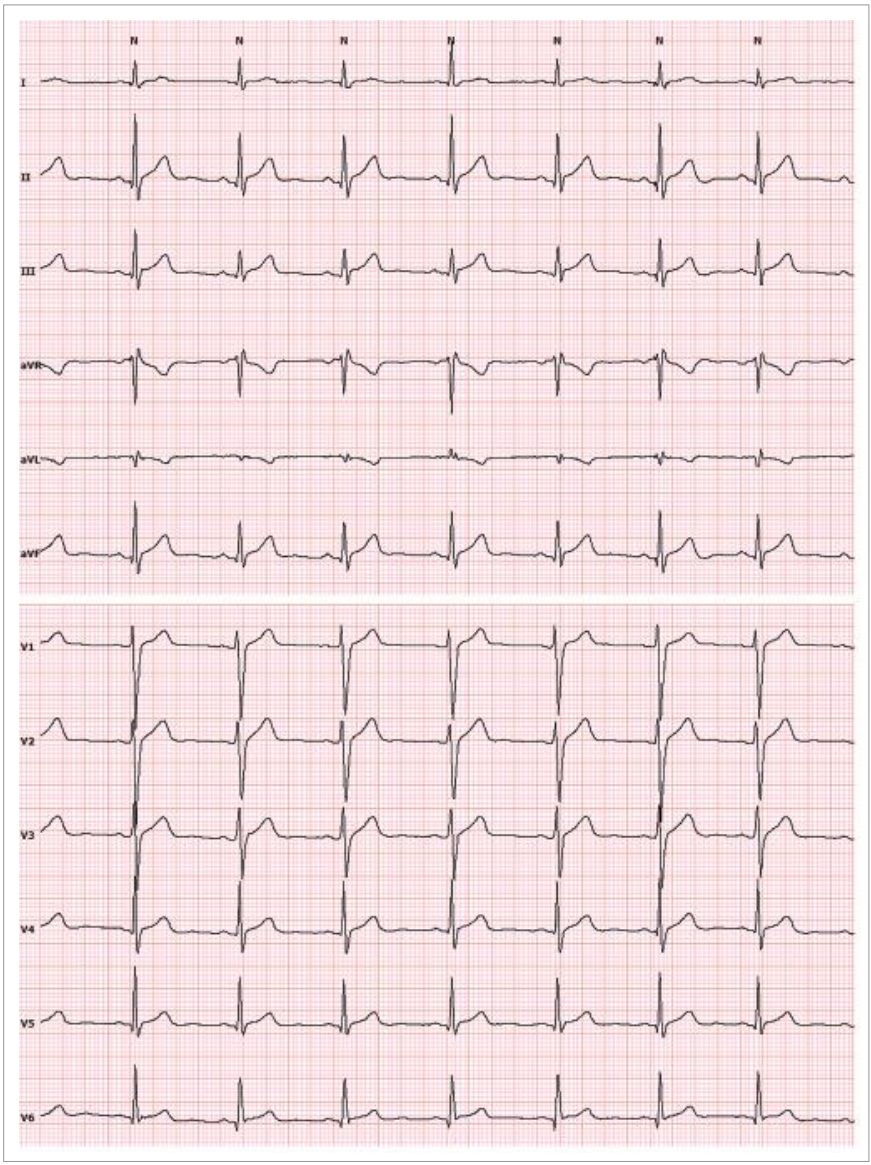

Electrocardiogram (ECG) showed sinus rhythm, heart rate 70 beats per minute, absence of ECG-criteria left ventricular hypertrophy and an improvement in repolarisation with normal $T$ waves.

\section{Discussion}

Hyperthyroidism can induce both haemodynamical and electrophysiological effects. Pathophysiological consequences of hyperthyroidism are mediated by triiodothyronine's (T3) genomic (binding to intranuclear specific receptor, acting as a transcriptional factor and promoting transcription of specific genes) and nongenomic actions (including direct cytoplasmatic effects not involving gene transcriptions). ${ }^{3}$ In cardiomyocytes, these actions lead to synthesis of calcium-activated ATPase, sarcoplasmic reticulum protein and phospholamban. ${ }^{3}$ T3 can also induce a remodulation of sodium, calcium and potassium channels with respective changes to intracellular ionic concentration. For example, Ma et al. demonstrated that in adult rats, $T 3$ administration is able to modify the expression of voltage-gated cardiac potassium channels, reducing the action potential duration both in the atrium and ventricle, although with a different response between the two districts. ${ }^{4}$

Thyroid hormones can also determine upregulation of beta-adrenergic receptors with increased sensitivity to catecholamine. ${ }^{3}$ As a consequence, there is an increase of chronotropy and inotropy, 3,5 which leads to increased heart rate, systolic blood pressure, stroke volume and cardiac output (cardiac preload is also augmented due to increased blood volume). Hyperthyroidism also reduces peripheral resistance, cardiac afterload and diastolic blood pressure. This complex condition may lead to the so-called 'high-output heart failure', which evolves from a hyperkinetic to a hypokinetic state and is characterised by dilated 
cardiomyopathy with reduced systolic ejection fraction. ${ }^{3,6,7}$ This can be either a reversible complication or a permanent condition.., 9

As we previously stated, hyperthyroidism also determines electrophysiological effects because of the influence on ion channels and the global positive chronotropic effect. In particular, this could be due to the different times of repolarisation of epi- and endo-myocardial cells that can be accentuated by beta-adrenergic action which abbreviates epi- and endo-cardiac potential action but not mid-cardial cells. ${ }^{10-2}$ As a result, patients with hyperthyroidism are at increased risk of premature beats, sinus tachycardia, atrial fibrillation, atrial flutter and impairment of QT interval (both short and long QT are possible). ${ }^{3.5,13}$

Bifid (or dome-and-dart or notched) $T$ waves are defined as an indented T wave with two distinguished peaks. ${ }^{14}$ The pathophysiological mechanism of bifid $T$ wave is still controversial. These alterations have been related to various conditions, either electrophysiological or anatomical, and could lead to inhomogeneous and local delayed ventricular repolarisation, generating a second component of $\mathrm{T}$ wave on the surface ECG. ${ }^{15}$ Bifid T waves seem to be more frequent in subjects with congenital cardiomyopathies. Awa et al. studied 249 children affected by various congenital heart diseases, and compared them to 520 healthy controls. They found a prevalence of $\mathrm{T}$ waves in approximately $85 \%$ of children with ventricular septal defects or patent ductus arteriosus, while they were present in only $32 \%$ of controls. ${ }^{16}$ Other conditions that, in the literature, have been associated with bifid T waves, albeit rarely, are congenital QT syndrome ${ }^{-17}$ class 3 antiarrhythmic medications, such as amiodarone; ${ }^{18}$ alcoholic cardiomyopathy: ${ }^{19}$ certain psychotropic and neuroleptics medications (i.e., phenothiazine); and cardiotoxic drugs, such as doxorubicin. ${ }^{20}$

As we previously illustrated, bifid T waves' pathophysiological origin is still not known, but seems to be related to anatomical and electrophysiological factors that induce alterations in ventricular repolarisation. In the first part of this discussion, we briefly reviewed the wide spectrum of hyperthyroidism effects on cardiovascular systems, highlighting the various structural and functional alterations, especially the influence of thyroid hormones on the basic generation of action potentials. All of these factors could be connected to the origin of bifid T waves in the case presented here. It is also worthwhile to note that the ECG alterations were not irreversible, since both signs of left ventricular enlargement and bifid T waves disappeared after restoring an euthyroid state. This finding has also been described by Lai et al., who reported the case of a 15-year-old boy with hyperthyroidism and similar ECG abnormalities. ${ }^{21}$ However, Lai et al. did not detect echocardiographic abnormalities in the patient, while we observed mild left ventricle dilatation, which improved after surgery (along with respective ECG signs). We hypothesise that dome-and-dart $\mathrm{T}$ wave abnormalities in hyperthyroidism could also reflect transient structural myocardial changes. Our data are in agreement with the recent study by Okosieme et al., which showed that effective treatment of hyperthyroidism is associated with lower cardiovascular events, compared to poorly controlled patients. ${ }^{22}$

In the management of Graves' disease, we follow international guidelines. In this patient both surgery and radioactive iodine were possible therapeutic strategies, without contraindications. ${ }^{2.23}$ We discussed the options with the patient, and he decided on the surgical approach, because he desired prompt control of the hyperthyroidism and to avoid exposure to radiation. We agreed with the patient's decision, and felt confident in his choice, because our hospital has an expert team of thyroid surgeons. ${ }^{2,23}$ One month after the intervention with treatment with $50 \mathrm{mcg}$ L-thyroxine per day, the patient was in a status of hypothyroidism, so the replacement therapy was increased to $75 \mathrm{mcg}$ per day. Notably, TSH was elevated, but not to levels observed in classic hypothyroidism. This could be related to the so-called 'phenomenon of hysteresis' that involves the hypothalamus-pituitary-thyroid axis; after the resolution of hyperthyroidism, the axis is described to have a sort of 'memory' that induces a delay in recovery after the TSH suppression caused by hyperthyroidism. ${ }^{24}$

In conclusion, hyperthyroidism should be considered among the differential diagnoses when T wave abnormalities on ECG and mild left ventricle dilatation on echocardiogram are observed. The correction of hyperthyroidism can reverse these abnormalities.
1. De Leo S, Lee SY, Braverman LE. Hyperthyroidism. Lancet. 2016;388:906-18.

2. Ross DS, Burch HB, Cooper DS, et al. 2016 American thyroid association guidelines for diagnosis and management of hyperthyroidism and other causes of thyrotoxicosis Thyroid. 2016;26:1343-421.

3. Vargas-Uricoeche $\mathrm{H}$, Sierra-Torres $\mathrm{CH}$. Thyroid hormones and the heart. Horm Mol Biol Clin Investig. 2014;18:15-26.

4. Ma ML, Watanabe $\mathrm{K}$, Watanabe $\mathrm{H}$, et al. Different gene expression of potassium channels by thyroid hormone and an antithyroid drug between the atrium and ventricle of rats. Jpn Heart J. 2003;44:101-10.

5. Klein I, Ojamaa K. Thyroid hormone and the cardiovascular system. N Engl J Med. 2001;344:501-9.

6. Boccalandro C, Boccalandro F, Orlander P, Wei CF. Severe reversible dilated cardiomyopathy and hyperthyroidism: case report and review of the literature. Endocr Pract. 2003;9:140-6.

7. Givertz MM, Mann DL. Epidemiology and natural history of recovery of left ventricular function in recent onset dilated cardiomyopathies. Curr Heart Fail Rep. 2013;10:1-17.

8. Al-Ghamdi AS, Aljohani N. Graves' thyrotoxicosis-induced reversible cardiomyopathy: a case report. Clin Med Insights Case Rep. 2013;6:47-50.
9. Londhey VA, Kamble US, Limaye CS, et al. Irreversible dilated cardiomyopathy due to thyrotoxicosis. I Assoc Physicians India. 2006:54:575-6

10. Yan $G X$, Antzelevitch C. Cellular basis for the normal T wave and the electrocardiographic manifestations of the long-QT syndrome. Circulation. 1998:98:1928-36.

11. Shimizu W, Antzelevitch C. Cellular basis for the ECG features of the LQT1 form of the long-QT syndrome: effects of beta-adrenergic agonists and antagonists and sodium channe blockers on transmural dispersion of repolarization and torsade de pointes. Circulation. 1998;98:2314-22.

12. Shimizu W, Antzelevitch C. Differential effects of beta-adrenergic agonists and antagonists in LQT1, LQT2 and LQT3 models of the long QT syndrome. J Am coll Cardiol. 2000;35:778-86.

13. Baladi IH, Rai AA, Ahmed SM. ECG changes in patients with primary hyperthyroidism. Pan Afr Med J. 2018;30:246.

14. Oyamada J, Shimizu C, Kim J, et al. Bifid T waves on the ECG and genetic variation in calcium channel voltage-dependent beta 2 subunit gene (CACNB2) in acute Kawasaki disease. Congenit Heart Dis. 2019;14:213-20.

15. Oka-Manabe S, Maruyama T, Urae R, et al. Prominent bifid T waves observed in the QT prolongation caused by complete atrioventricular blockade in a hypokalemic diabetic patient. J Electrocardiol. 1999;32:289-92.

16. Awa $\mathrm{S}$, Linde LM, Oshima M, et al. The significance of late-phased dart T wave in the electrocardiogram of children. Am Heart I. 1970:80:619-28.

17. Lehmann MH, Suzuki F, Fromm BS, et al. T wave "humps" as a potential electrocardiographic marker of the long QT syndrome J Am Coll Cardiol. 1994:24:746-54.

18. Pollack PT, Sharma AD, Carruthers SG. Correlation of amiodarone dosage, heart rate, QT interval and corneal microdeposits with serum amiodarone and desethylamiodarone concentrations. Am J Cardiol. 1989;64:1138-43.

19. Priest RG, Binns JK, Kitchen AH. Electrocardiogram in alcoholism and accompanying physical disease. Br Med J. 1966;1:1453-5.

20. Chen WC, Zeng ZR, Wu CF. Clinical significance of bifid T waves. Jpn Circ J. 1986;50:232-7.

21. Lai P, Yuan JL, Xue JH, Qiu YQ. Hyperthyroidism with dome-and-dart T wave: a case report: a care-compliant article. Medicine (Baltimore). 2017:96:e6060.

22. Okosieme OE, Taylor PN, Evans C, et al. Primary therapy of Graves' disease and cardiovascular morbidity and mortality: a linked-record cohort study. Lancet Diabetes Endocrinol. 2019;7:278-87.

23. Kahaly GJ, Bartalena L, Hegedüs L, et al. 2018 European Thyroid Association guideline for the management of Graves' hyperthyroidism. Eur Thyroid J. 2018;7:167-86.

24. Leow MK. A review of the phenomenon of hysteresis in the hypothalamus-pituitary-thyroid axis. Front Endocrinol (Lausanne). 2016;7:64. 